\title{
Off-Design Considerations through the Properties of Some Pressure-Ratio Line of Radial Inflow Turbines
}

\author{
N. Binder, X. Carbonneau, and P. Chassaing \\ Institut Supérieur de l'Aéronautique et de l'Espace (ISAE), Université de Toulouse, 10 Avenue Edouard Belin, \\ 31055 Toulouse Cedex 4, France \\ Correspondence should be addressed to N. Binder, nicolas.binder@isae.fr
}

Received 18 July 2008; Accepted 17 November 2008

Recommended by Gerard Bois

\begin{abstract}
Radial turbines are commonly used in applications involving operation through severe off-design conditions. The emergence of variable-geometry systems leads to the distinction between two off-design concepts: operational and geometric off-designs. Both of these operating constraints should be integrated in the design procedure. Recent developments in prediction and optimization methods allowed such an integration, but involving complex algorithms is coupled with semiempiric loss models. This paper provides a basis to obtain simple information from an existing or predesigned machine, for both operational and geometric offdesign conditions. An alternative turbine map is defined using loading and flow coefficients. A one-dimensional analysis shows that the constant pressure-ratio lines are straight lines whose slope is remarkably correlated with the pressure-ratio value and geometrical characteristics. This theoretical approach is validated against the experimentation of two machines, the linearity is observed in both cases. The direct influence of the stator configuration on the pressure-ratio lines confirms the applicability of this work to variable-geometry stages. A dimensionless cross-section of the stator is thus defined. However, the unexpected displacement of the intercept of the pressure-ratio lines limits the application field of this method. Nevertheless, a simple performance prediction analysis is proposed for blocked mass flow operation.
\end{abstract}

Copyright ( $) 2008$ N. Binder et al. This is an open access article distributed under the Creative Commons Attribution License, which permits unrestricted use, distribution, and reproduction in any medium, provided the original work is properly cited.

\section{INTRODUCTION}

Nowadays radial-inflow turbines are preferred to axial machines in an important number of applications. Their low-cost manufacturing and compactness are appreciated in fields such as turbocharging, air-conditioning systems, or cryogenics. In addition to those industrial qualities, radialturbine stages have interesting operating abilities. Compared to axial geometries, the peak efficiency is generally lower, and the stage integration does not ease multistage functioning. But the radius change across the rotor gives a positive contribution in the Euler equation, resulting in higher specific power. This balance is thoroughly discussed in [1] where charts are presented, based on dimensionless parameters of the design point (specific diameter and speed). Regions of best efficiency are defined for the different architectures, and first-design rules are proposed. However, this does not warrant an adequate off-design performance. Actually the compactness of the impeller of a radial turbine allows very high rotational speed, and the stage can sustain high pressure ratios. This flexibility is appreciated in case of off-design functioning necessity. Even for such cases, the design of the machines has long been focused on a single specification, the performance range being evaluated subsequently. Numbers of efficient procedures derived from one-dimension analysis have been proposed with that purpose, for example, by Rohlik [2], Rogers [3], or Whitfield and Baines [4]. Those procedures improved in time with accurate loss, deviation, and blockage models based on physical considerations, and supported by data over a large number of experiments. See, for instance, the reviews by Moustapha et al. [5], Aungier [6], or Ebaid et al. [7] where a unified approach is proposed. These procedures now involve elaborated codes allowing off-design predictions, made so far with the use of specific routines such as Wasserbauer and Glassmann [8]. The improvement of CFD has given the opportunity to analyze a stage at minimal cost at the end of the design procedure. The use of optimization algorithms also becomes accessible. 


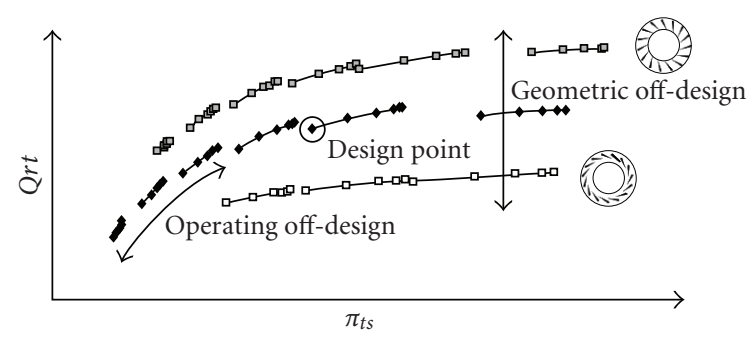

FIGURE 1: Off-design configurations.

For example, Harinck et al. [9] optimized a radial turbine by means of genetic algorithm, and Demeulenaere and Hirsh [10] proposed a multipoint optimization procedure to insure best off-design performances. All these methods are efficient and give accurate results, but require a specific involvement of the users for their implementation.

This assessment gets more complex when considering variable-geometry stages. The operating range of such stages is widened, by adapting the flow capacity to the inlet conditions. Thus in addition to "operating off-design" the geometry variations shift the stator from its design configuration, creating a "geometric off-design" (Figure 1). Its effect on classical design-parameters has been presented in [11]. Apart from specific losses due to technological effects (mechanical clearance, partial admission at rotor inlet, spacers), the nozzle configuration is likely to take extreme positions, which may prove critical when using classical models.

The present paper provides a basis to obtain simple offdesign information from existing or predesigned machines on either fixed- or variable-geometry stages. It is based on an alternative presentation of the turbine map which gives a new insight into the global functioning of the machine and unifies the influences of operational and geometric off-designs. This observation has led to introduce a one-dimension analysis whose hypotheses are not based on design-point simplification. The alternative diagram is detailed in the first part of this paper, through the properties of a pressureratio line. The results of the analysis will be compared with experimental results for validation in the second part. In the last part, first applications for design and prediction are proposed.

\section{THE PRESSURE-RATIO LINE}

The choice of aero-thermodynamic parameters used to describe turbomachines has been long discussed in the literature (see, e.g., $[12,13])$. Similarity analysis shows that this description can be summarized by the evolution of four nondimensional parameters if the Reynolds number and gas effects are neglected. The classic representation of the operating field of compressor or turbine stages is given in a map of reduced mass flow against pressure ratio, for reduced-speed lines and efficiency regions. An alternative is found using other nondimensional quantities such as the flow and loading coefficients. Those quantities are currently used during the first steps of design, to obtain the main characteristics of the stage through correlations (see [14]), but can easily be expressed as combination of the classic parameters. Consequently a $\psi-\phi$ map should express more than correlations, and actually presents interesting characteristics. More specifically, a one-dimension approach gives a simple equation for the trajectory of a constant pressure-ratio line in this map. This analysis is presented as follows.

To ease the integration of the Euler equation at the inlet and outlet of the rotor, two hypotheses on the flow are generally considered as follows:

(i) the homogeneity of the flow along the span of the blade at the inlet;

(ii) the conservation of the angular moment at the outlet.

Thus, considering the first law of thermodynamics, the Euler equation gives for total enthalpy variation:

$$
\Delta H=U_{4} V_{\theta 4}-U_{5}\left(U_{5}-W_{\theta 5}\right)
$$

The second hypothesis insures the possibility to write this equation at any rotor outlet radius. No clear convention is adopted in the literature even if the RMS radius is often chosen. The outlet tip radius will here be considered in order to preserve the expression of the global trim of the rotor in the equations. The above expression can then be written as follows:

$$
\frac{\Delta H}{U_{4}^{2}}=\frac{\sqrt{r T_{i 1}}}{U_{4}}\left(\frac{V_{\theta 4}}{\sqrt{r T_{i 1}}}+\frac{D_{5}}{D_{4}} \frac{W_{\theta 5}}{\sqrt{r T_{i 1}}}\right)-\left(\frac{D_{5}}{D_{4}}\right)^{2} .
$$

Considering isentropic process in the stator, conservation of mass at rotor inlet gives

$$
\frac{V_{\theta 4}}{\sqrt{r T_{i 1}}}=\frac{\dot{m} \sqrt{r T_{i 1}}}{P_{i 1}} \frac{\tan \left(\alpha_{4}\right)}{S_{4}}\left(\frac{P_{i 1}}{P_{4}}\right)^{1 / \gamma} .
$$

Considering total to static efficiency definition, conservation of mass at the rotor outlet gives

$$
\begin{aligned}
\frac{W_{\theta 5}}{\sqrt{r T_{i 1}}}= & \frac{\dot{m} \sqrt{r T_{i 1}}}{P_{i 1}} \frac{\tan \left(\beta_{5}\right)}{S_{5}} \pi_{t s} \\
& \times\left\{1-\eta_{t s}\left[1-\left(\frac{1}{\pi_{t s}}\right)^{(\gamma-1) / \gamma}\right]\right\}\left(\frac{P_{i 5}}{P_{5}}\right)^{(1-\gamma) / \gamma} .
\end{aligned}
$$

Equations (2), (3), and (4) give

$$
\begin{aligned}
\frac{\Delta H}{U_{4}^{2}}=\frac{r T_{i 1} \dot{m}}{U_{4} P_{i 1} D_{4}^{2}}\{ & {\left[\frac{P_{i 1}}{P_{4}}\right]^{1 / \gamma} \frac{D_{4}^{2} \tan \left(\alpha_{4}\right)}{S_{4}} } \\
& +\left[\frac{P_{i 5}}{P_{5}}\right]^{(1-\gamma) / \gamma} \frac{D_{5}}{D_{4}} \frac{D_{4}^{2} \tan \left(\beta_{5}\right)}{S_{5}} \pi_{t s} \\
& \left.\times\left[1-\eta_{t s}\left[1-\left(\frac{1}{\pi_{t s}}\right)^{(\gamma-1) / \gamma}\right]\right]\right\}-\left(\frac{D_{5}}{D_{4}}\right)^{2} .
\end{aligned}
$$


Loading and flow coefficients are defined as follows:

$$
\begin{gathered}
\psi=\frac{\Delta H}{U_{4}^{2}}, \\
\phi=\frac{r T_{i 1} \dot{m}}{U_{4} P_{i 1}(\pi / 4) D_{4}^{2}} .
\end{gathered}
$$

For simplicity's sake, we will also define an intermediate pressure ratio and two geometric factors:

$$
\begin{gathered}
\xi=\frac{P_{5}}{P_{4}}, \\
S_{4}^{*}=\frac{S_{4}}{D_{4}^{2} \tan \left(\alpha_{4}\right)}, \\
S_{5}^{*}=\frac{S_{5}}{D_{4}^{2} \tan \left(\beta_{5}\right)} .
\end{gathered}
$$

The use of the sine rule to estimate $\alpha_{3}$ and the assumption of small changes of this angle through the free space allows one to express $S_{4}^{*}$ after the stator characteristics at the throat:

$$
S_{4}^{*}=S_{3}^{*}=\frac{S_{3}}{D_{4}^{2}\left(r_{3} / r_{4}\right) \sin \left(\alpha_{3}\right)} .
$$

$S_{3}^{*}$ and $S_{5}^{*}$, with these definitions, are nondimensional parameters characterizing both the stator and rotor geometries and can be considered as dimensionless cross-sections. In that condition (5) becomes as follows:

$$
\begin{aligned}
\psi=\phi \cdot \frac{\pi}{4}\{ & {\left[\xi \cdot \pi_{t s}\right]^{1 / \gamma}\left(S_{3}^{*}\right)^{-1}+\left[\frac{P_{i 5}}{P_{5}}\right]^{(1-\gamma) / \gamma} \frac{D_{5}}{D_{4}}\left(S_{5}^{*}\right)^{-1} } \\
& \left.\times \pi_{t s}\left[1-\eta_{t s}\left[1-\left(\frac{1}{\pi_{t s}}\right)^{(\gamma-1) / \gamma}\right]\right]\right\}-\left(\frac{D_{5}}{D_{4}}\right)^{2} .
\end{aligned}
$$

At constant pressure ratio, small variations of $\xi$ are expected. The influences of $\eta_{t s}$ and $P_{i 5} / P_{5}$ can be shown to be negligible compared with those of $\pi_{t s}, S_{3}^{*}$ and $S_{5}^{*}$. Therefore, the trajectory of a pressure-ratio line in a $\psi-\phi$ map, for a given geometry, is expected to be linear. The intercept is fixed and depends on the trim of the rotor. The slope increases with the pressure ratio and decreases with $S_{3}^{*}$ and $S_{5}^{*}$ as illustrated in Figure 2(a).

In this diagram, the effects of operating and geometric off-designs are unified. Both are reflected by the displacement of the operating point along the pressure-ratio line, or the modification of its slope. Other properties of the $\psi-\phi$ diagram can be further detailed in order to locate classic features as speed lines and best-efficiency regions. The flow coefficient is then written using reduced rotational speed and mass flow, the loading coefficient using efficiency and reduced tip speed:

$$
\begin{gathered}
\phi=\frac{240}{\pi^{2}} \frac{Q r t}{N r t}, \\
\psi=\frac{1}{2} \eta_{t s}\left(\frac{C_{s}}{U_{4}}\right)^{2} .
\end{gathered}
$$

Consequently the shape of the speed lines can easily be deduced. For a given value of $N r t, \phi$ has the same variation as

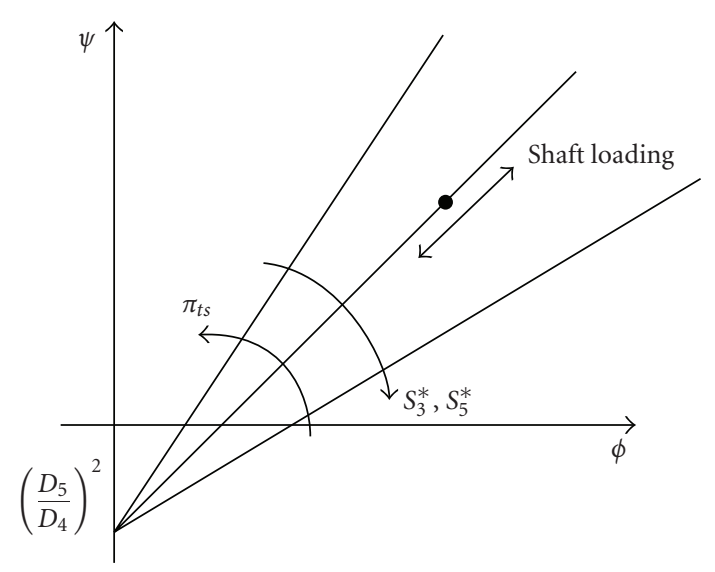

(a)

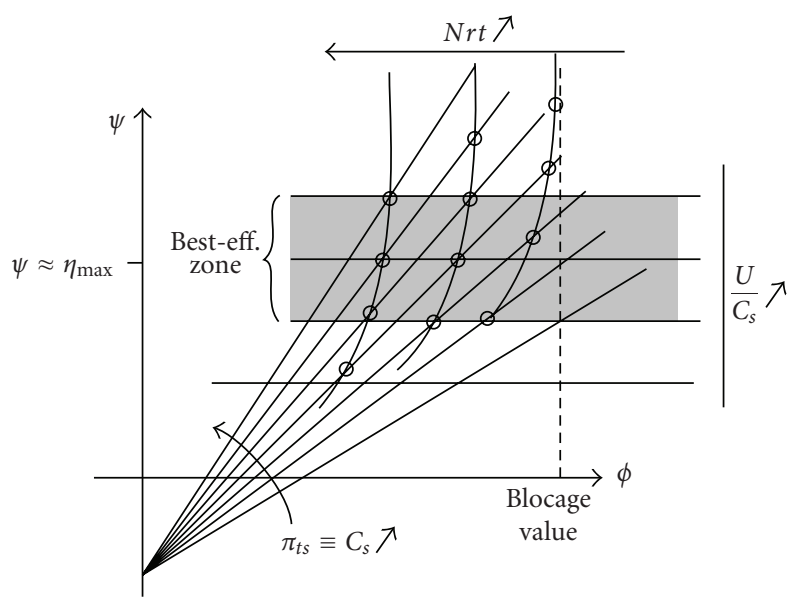

(b)

Figure 2: $\psi-\phi$ map.

Qrt and will increase with pressure ratio. This is observed at each intersection with a pressure-ratio line, until the choking value is reached. As Nrt increases, the choking value of $\phi$ decreases, hence defining the stack of speed lines quoted on Figure 2(b). Besides, for fixed inlet temperature, pressureratio lines are also adiabatic-speed lines by definition. An intersection between a pressure-ratio line and a speed line defines the value of the reduced tip speed $\left(U_{4} / C_{s}\right)$. Assuming small variations of efficiency when $U_{4} / C_{s}$ is fixed, relation (11) insures the conservation of this value along a $\psi$ line. Considering that best efficiency is reached near $U_{4} / C_{s}=0.7$, the best-efficiency region can be expected at $\psi \approx \eta_{\max }$ as illustrated in Figure 2(b).

The potential of the $\psi-\phi$ diagram has therefore been demonstrated. This alternative turbine map represents offdesign behavior in a quite simple transcription. Moreover, it is theoretically possible to model the whole performance of a stage with straight lines, from its predesign characteristics, including influence of nozzle modifications for variablegeometry stages. These properties are obtained subject to restrictive hypothesis of a one-dimension approach and have 
TABLE 1

\begin{tabular}{lcc}
\hline & TS1 & TS2 \\
\hline$D_{4}(\mathrm{~mm})$ & 141 & 47 \\
$N(\mathrm{rpm}$ at design $)$ & 45000 & 150000 \\
$\left(D_{5} / D_{4}\right)^{2}$ & 0.32 & 0.67 \\
$S_{3}^{*}$ & 0.038 & 0.074 to 0.52 \\
$S_{5}^{*}$ & 0.57 & 0.91 \\
\hline
\end{tabular}

thus to be confronted to real operation. Experimental results of two turbine stages will now be analyzed in that context, focusing on the influence of the pressure ratio for the first stage and stator geometry for the second.

\section{EXPERIMENTAL VERIFICATION}

The above analysis is compared to the results of experiments performed with two different turbine stages, representing either operational or geometric off-designs. The properties of the pressure-ratio lines in a $\psi-\phi$ diagram have been presented previously. According to relation (9), the slopes of the lines are strongly linked to the pressureratio value and both the dimensionless sections of the stator and the rotor. As the rotor geometry is obviously fixed when functioning, the influences of the first two parameters only will be checked. The first turbine stage (TS1) is part of an aircraft air-conditioning pack. It has been tested for a wide range of pressure-ratio values. The second one (TS2) is a variable-geometry turbocharger turbine in which the modification of blade's angle of attack allows variations of the dimensionless stator section.

The characteristics of the stages are presented in Table 1, which together with (9) gives the expected pressure-ratio lines for the two stages. The values of the efficiency and intermediate pressure-ratio $\left(\eta_{t s}\right.$ and $\left.\xi\right)$ are kept constant for each stage and set according to the design specification. Four values of $\pi_{t}(1.8,2.5,3$, and 3.5) are considered for TS1, three opening positions at $\pi_{t}=1.6$ for TS2 (Figure 3 ).

As relation (11) imposes the order of magnitude of $\psi$ for an efficient operation, a tenfold difference is observed between the values of $\phi$ for the two stages. The initial stage specification and the choice of the rotor diameter set this proportion. Consequently, the slopes of the pressure-ratio lines are quite different from one stage to another $(\approx 40$ for TS1; $\approx 5$ for TS2). As a result, the dimensionless stator section $\left(S_{3}^{*}\right)$ also presents large differences (Table 1). Relation (9) actually determines the correlation between the slope of pressure-ratio lines and the dimensionless stator section, thus expressing the link between operating behavior and geometry configuration of the stage.

The experimental results of these two stages are now considered. The four dimensionless parameters (Qrt, Nrt, $\pi_{t}$, and $\eta$ ) are obtained through mass flow, pressure, and temperature-drop measurements. Rotational speed is given by count on the compressor stage; the values of

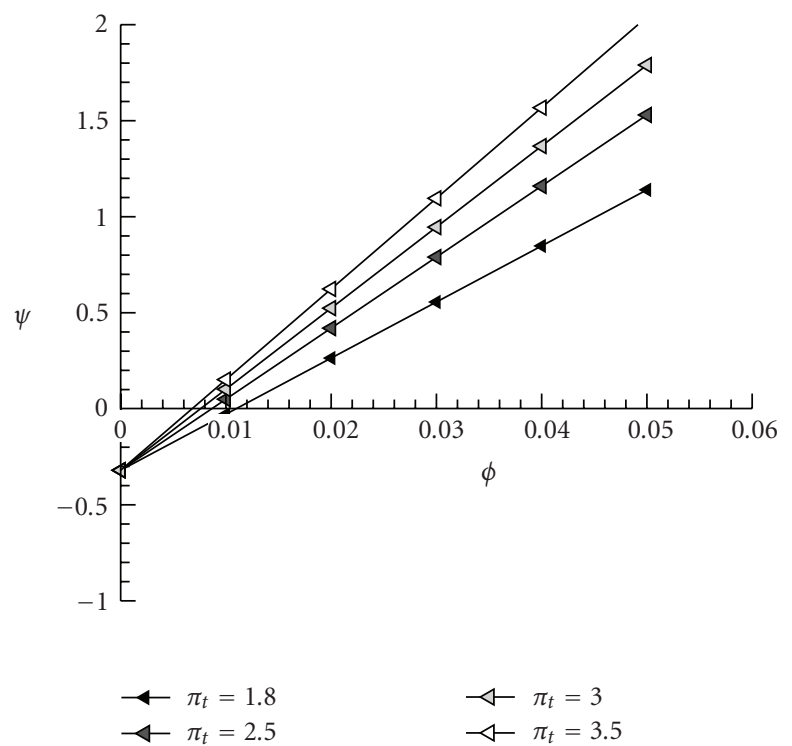

(a)

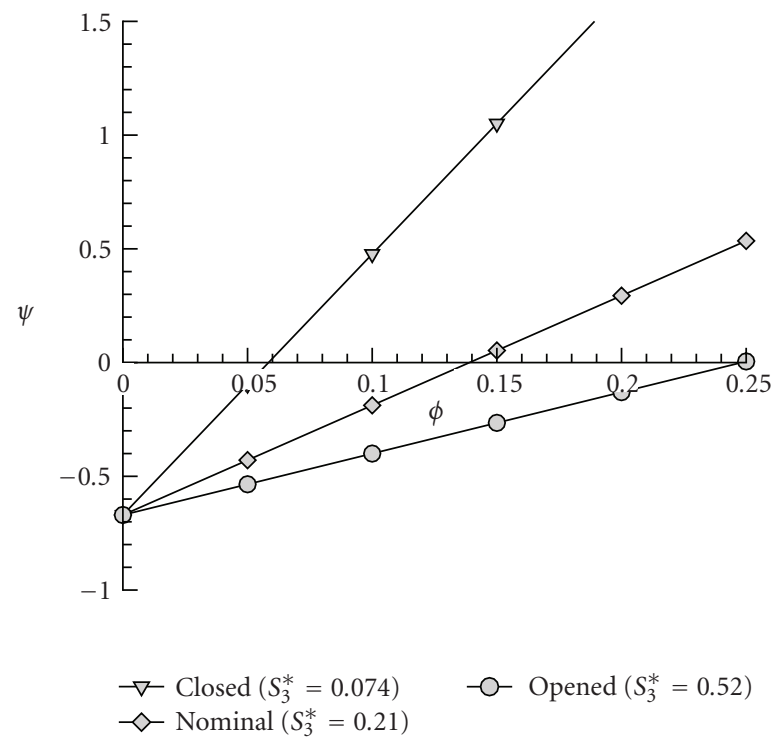

(b)

Figure 3: Pressure-ratio line prediction for (a) TS1 and (b) TS2.

$\psi$ and $\phi$ follow. To avoid perturbation of temperaturedrop measurement by conduction, tests were conducted under ambient inlet temperature conditions. For detailed information about the experimental facilities see [15] for TS1, and [11] for TS2. The results are plotted in the $\psi-\phi$ map (see Figure 4).

The linearity of the evolution is remarkable. It supports the initial assumption according to which the influence of $\eta_{t s}$ and $\xi$ variations along the line is small. The expected influences of pressure ratio and $S_{3}^{*}$ on the slope of the line are confirmed.

The magnitude of the gradient is consistent with what was expected in Figure 3. On the contrary, the displacement 


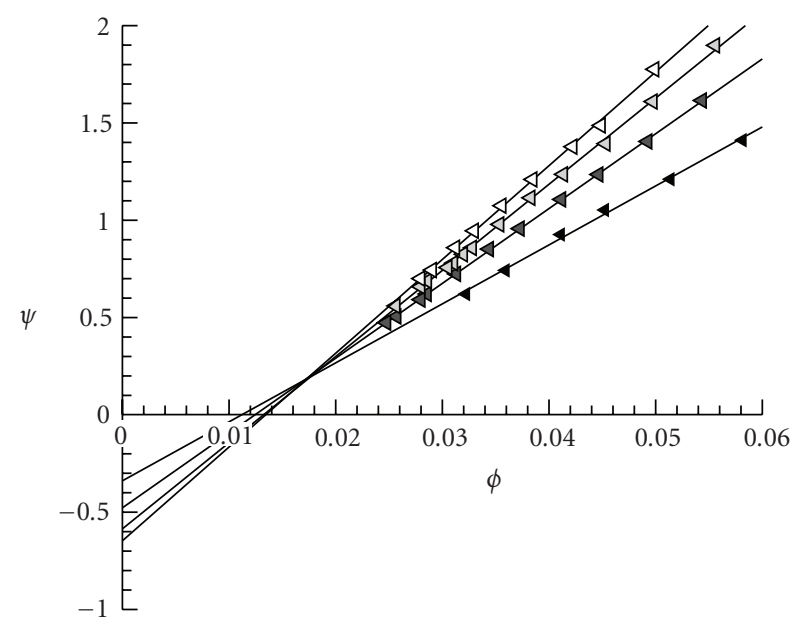

$$
\begin{array}{ll}
\longleftarrow \pi_{t}=1.8 & \triangleleft \pi_{t}=3 \\
\longleftarrow-\pi_{t}=2.5 & \checkmark ヶ \pi_{t}=3.5
\end{array}
$$

(a)

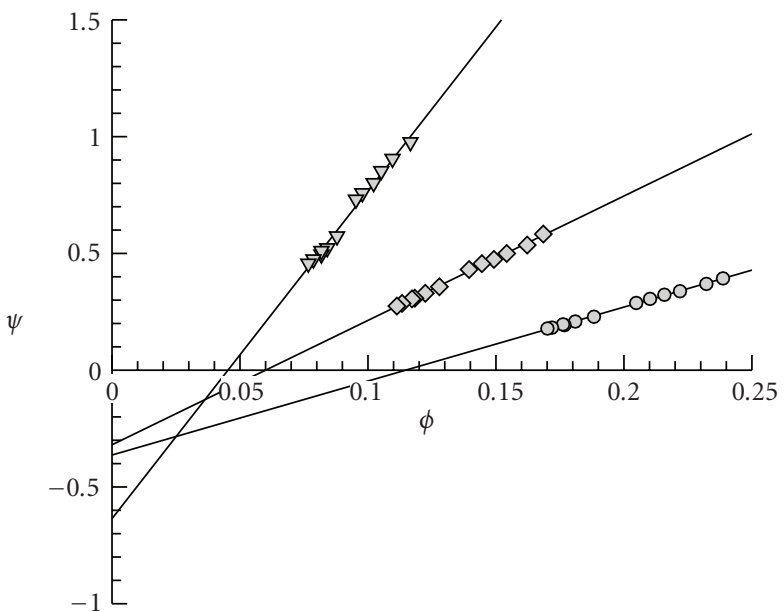

$$
\rightarrow-\text { Closed }\left(S_{3}^{*}=0.074\right) \quad-0-\text { Opened }\left(S_{3}^{*}=0.52\right)
$$$$
\checkmark \text { Nominal }\left(S_{3}^{*}=0.21\right)
$$

(b)

Figure 4: Experimental pressure-ratio line for (a) TS1 and (b) TS2.

of the intercept along the axis is unexpected. According to (9), it was supposed to be fixed by the rotor geometry, with no possible evolution. The origin of this mismatch should be found in the second hypothesis considered for the integration of Euler equation presented in the first part of the paper. Actually, the angular momentum is not conserved at the outlet of a turbine stage, particularly in off-design conditions. Five-hole-probes measurements are available for the stage TS1. An analysis of these results should give indications of the correction to bring, and help to choose the correct outlet radius considered for the integration.

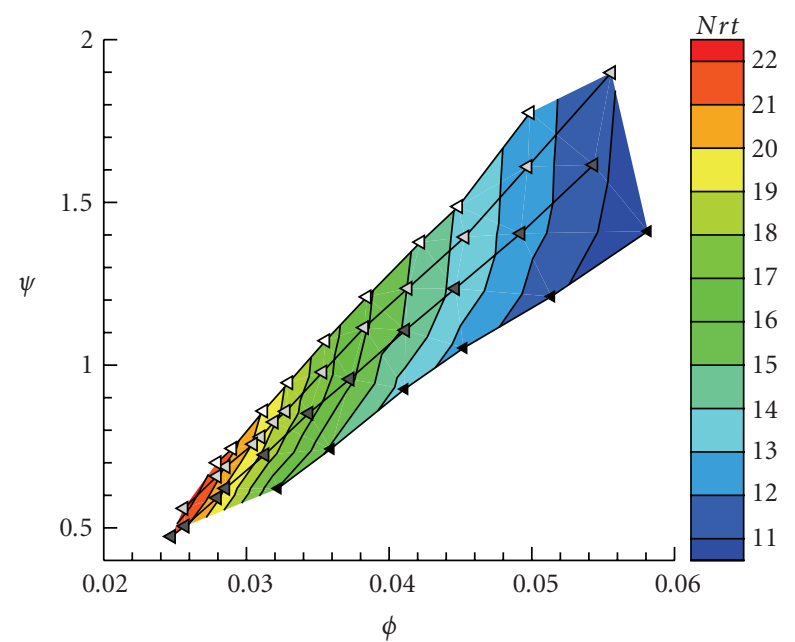

(a)

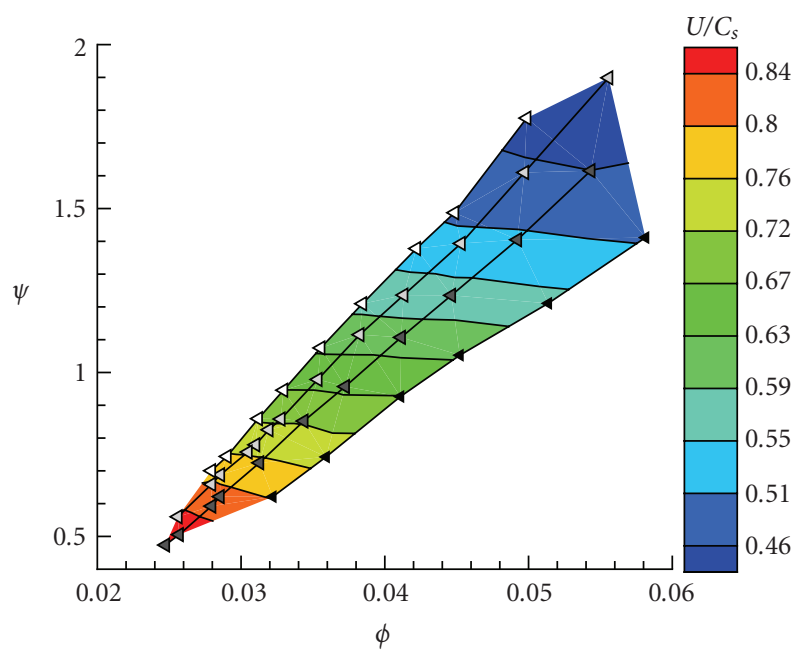

(b)

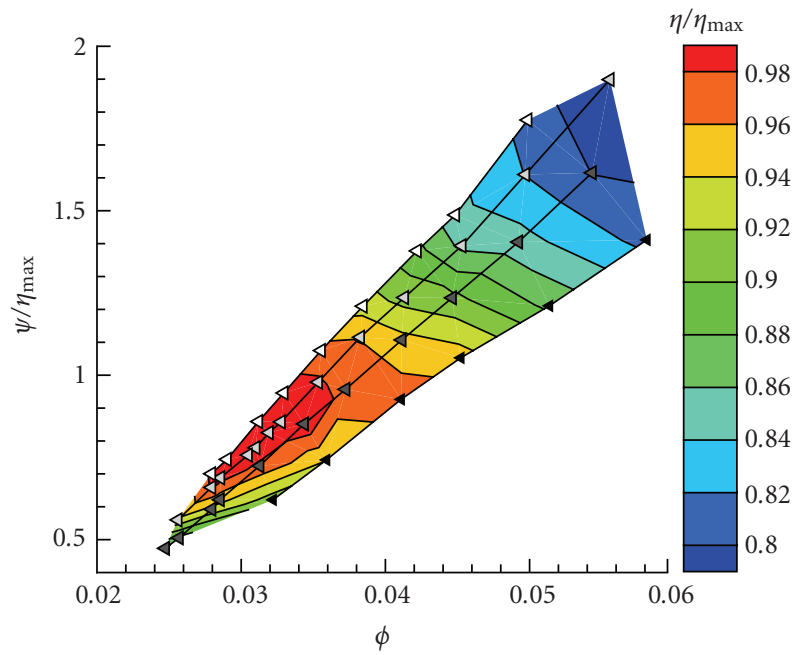

(c)

Figure 5: (a) Interpolation of speed, (b) reduced speed, and (c) efficiency lines. 
The location of other characteristic lines completes this verification. Speed, reduced tip speed, and efficiency lines of the stage TS1 are interpoled on the diagram (Figures 5(a), $5(\mathrm{~b})$, and 5(c)). The shape of the speed lines is confirmed with respect to Figure 2(b). The reduced tip-speed lines do not strictly coincide with $\psi$ lines due to effective efficiency variations. Despite these variations, the best-efficiency region is found as expected at $\psi \approx \eta_{\text {max }}$.

This experimental verification leads to a partial validation of the one-dimension analysis. The pressure-ratio lines are actually straight lines in a $\psi-\phi$ map. The unification of geometric and operational off-design transcriptions has been observed. The best-efficiency regions are found where expected. However, the displacement of the intercept was unexpected. It actually needs more attention. At this state this phenomenon limits the predictive potential of the diagram. Still, applications of this work can now be considered, in particular a first approach to design considerations and to performance predictions.

\section{APPLICATION FOR DESIGN AND PREDICTION}

\subsection{Design}

The first application of this work concerns the design of vaned nozzles. Equation (9) shows the importance of the stator dimensionless section $S_{3}^{*}$. In a one-dimension approach, the conservation of its value when changing the stator configuration should not influence the stage behavior (either at design point or at off-design conditions). The designer is free to define the right set of radii, outlet angle, and geometric section for best efficiency, using classical criteria (such as found, e.g., in [16]). Blockage, deviation, and losses will certainly affect this assessment, but most likely in a lesser measure. This point will be discussed in a forthcoming paper.

If conservation of $S_{3}^{*}$ preserves the stage behavior, its variation logically modifies the operating characteristics of the stage. Through this modification, adaptation of the stage to inlet changing conditions is possible, thus defining the "variable-geometry" concept. $S_{3}^{*}$ is therefore identified as the determining parameter when variable-geometry effects are considered.

Finally, for fixed-geometry stages, an early evaluation of $S_{3}^{*}$ should prove useful to estimate off-design requirements feasibility. In design process, the value of loading coefficient is set in order to maximize efficiency, according to relation (11). The specification and the choice of inlet diameter of the rotor will determine the flow-coefficient value at the design point. This choice can be conducted for performance (Chen and Baines [14] recommend 0.2-0.4 for better peak efficiency) or be constrained by mechanical or economic limitations (some designers may want to use existing basis for the new specification). The order of magnitude for the slope of pressure-ratio line of the stage is then frozen and (9) can give a first evaluation of the stator configuration through $S_{3}^{*}$. This value allows a first estimation of the stage's ability to reach specific operating points.

\subsection{Prediction}

A second application lies in prediction of performances. As straight lines, pressure-ratio lines are easily modelled in the $\psi-\phi$ map. But the displacement of the intercept presented in the second part of this paper coupled with influence of deviation, blockage, and losses forbids the full prediction of the $\psi$ - $\phi$ diagram. However, the interpolation of the complete map should be possible with two points of each pressure-ratio line. At this point the difficulty lies in the back deduction of the four classical dimensionless parameters ( $Q r t, N r t, \pi_{t}$, and $\eta$ ). The following two cases are considered.

(i) Choking is not reached: four parameters have to be determined, out of three equations based on the knowledge of $\psi$ or $\phi, \pi_{t}$ and the pressure-ratio line equation. The problem is open and cannot be solved simply.

(ii) Choking is reached or small variations of mass flow are expected: Qrt is frozen, removing the need of an extra equation. Two operating points of a pressure-ratio line allow the prediction of the whole performance line.

In the first case, a separation procedure is being developed and needs more attention. For the second case, the evolution of efficiency against rotational speed can be deduced. Rewriting (11) with $N r t, \pi_{t}$, and $\eta$, and using the definition (10) along a pressure-ratio line trajectory, the following equation can be established:

$$
\frac{\eta}{N r t^{2}}=A \cdot \frac{Q r t}{N r t}+B
$$

where $A$ and $B$ are determined after the pressure-ratio line constants and the value of $\pi_{t}$. Thereby the evolution of efficiency against rotational speed at constant pressure ratio and constant mass flow is quadratic. An example is proposed in Figure 6. Three pressure-ratio lines (one for TS1, and two for TS2 in closed-nozzle configuration) are presented; small mass flow variations are observed (Figure 6(a)). Two points of each line are selected, giving the coefficients of the $\psi-\phi$ line (Figure 6(b)). Equation (12) expresses the expected evolution of efficiency against rotational speed, the prediction and the results are plotted on Figure 6(c).

A very good agreement is achieved between the predicted interpolation and the actual measurements. The evolutions of rotational speed are reproduced for both stages, and the influence of pressure ratio for TS2 is consistent. The conservation of the mass flow along the pressure-ratio line has direct influence on the accuracy. Thereby, the prediction is much better for stage TS1 at blockage than for TS2 where blockage is not reached. But, in both cases, it has been possible to obtain the whole pressure-ratio line from only two operating points. This process is likely to reduce the number of points necessary to obtain the full map of a stage. 


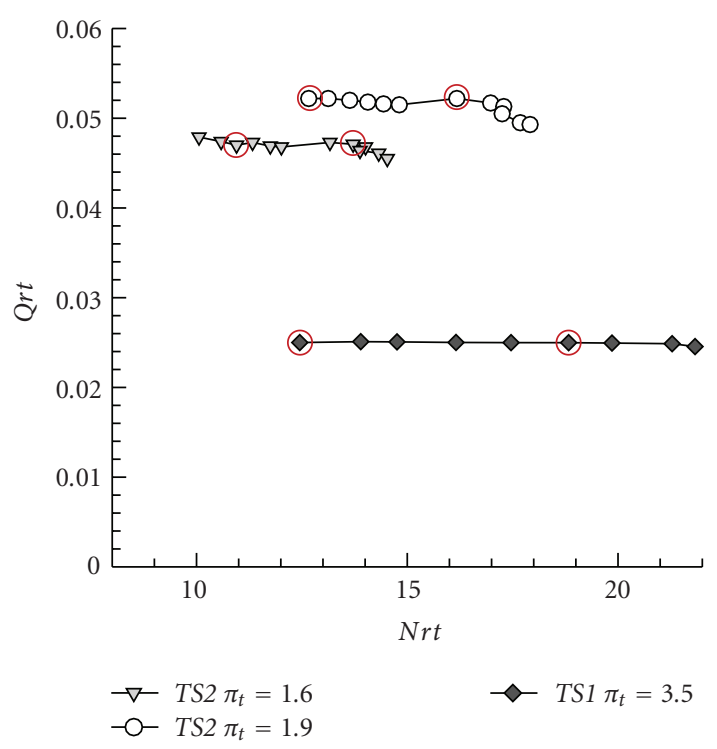

(a)

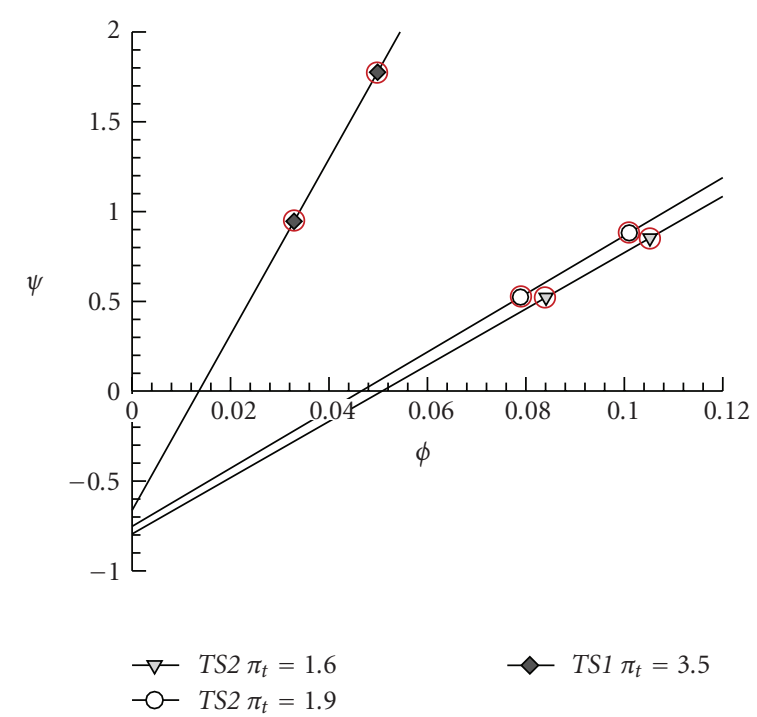

(b)

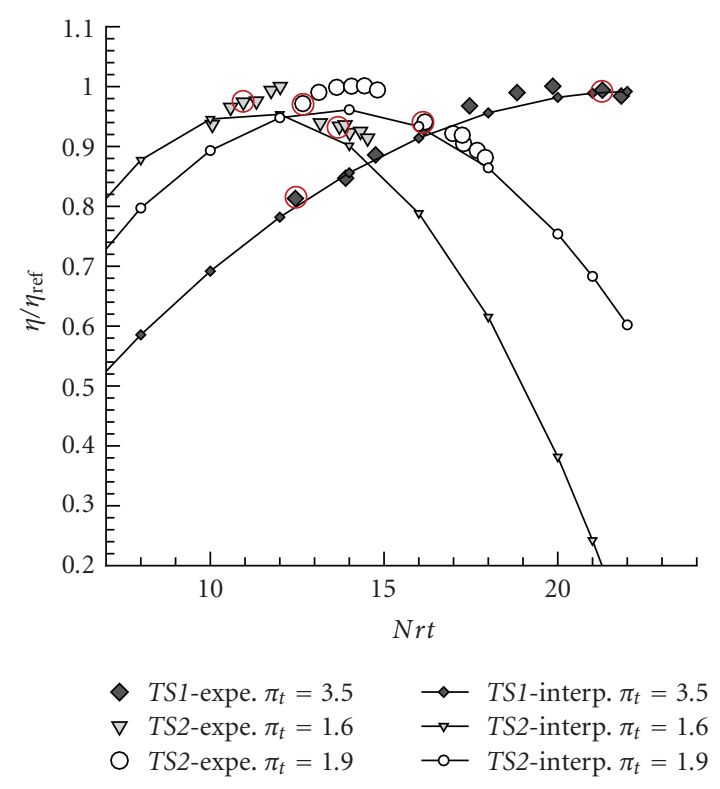

(c)

FIGURE 6: Interpolation process verification.

\section{CONCLUSION}

A new definition of a turbine map has been presented. Based on the properties of the pressure-ratio lines, this alternative map is supported by a one-dimension analysis and experimental verifications. This diagram not only allows simple representation of the stage behavior through straight lines, but also unifies geometric and operational off-design effects. The slope of the pressure-ratio lines is significantly influenced by the pressure-ratio itself and by a dimensionless parameter for characterizing the stator. Experimental results for two stages showed similar influences in good agreement with the analysis. However, the intercept of the line is not fixed as was expected. More work is currently conducted to better understand this effect and take it into account. The importance of the stator dimensionless cross-section has been shown. Its fundamental role in the definition of stage behavior has been assessed in the case of variable-geometry devices, for which it proved particularly well adapted. Finally, a first application to the prediction of performances has been performed for choked configurations. In this case, it has been shown that a full pressure-ratio line can accurately be obtained from two of its points. Forthcoming work will focus on three axes: (i) the influence of dimensionless stator section and its applications in a variable-geometry context, (ii) the influence of rotor outlet flow on the displacement of the intercept of the pressure-ratio line, (iii) the setup of a nonrestrictive interpolation process for nonchocked stage functioning. 


\section{NOMENCLATURE}

\section{NOTATION}

$C_{s}$ : Isentropic speed

D: Diameter

$H$ : Enthalpy

in: Mass flow

Nrt: Reduced rotational speed

$P: \quad$ Static pressure

$P_{i}$ : Total pressure

Qrt: Reduced mass flow

$U$ : Tip speed

$V:$ Absolute velocity

$W$ : Relative velocity

$r$ : Ideal gas constant

$S$ : Geometric cross-section

$T$ : $\quad$ Static temperature

$T_{i}$ : Total temperature

$\alpha$ : Absolute angle

$\beta$ : Relative angle

$\gamma$ : Specific heat ratio

$\eta$ : Efficiency

$\xi$ : $\quad$ Partial pressure ratio

$\pi_{t}$ : Turbine pressure ratio

$\phi$ : $\quad$ Flow coefficient

$\psi$ : Loading coefficient

\section{SUBSCRIPT}

1: Stage inlet

2: Stator inlet

3: Stator throat

4: Rotor inlet

5: Rotor outlet

$\theta$ : Tangential component

ts: Total to static reference

\section{REFERENCES}

[1] O. E. Balje, Turbomachines: A Guide to Design, Selection and Theory, John Wiley \& Sons, New York, NY, USA, 1981.

[2] H. E. Rohlik, "Analytical determination of radial inflow turbine design geometry for maximum efficiency," Tech. Rep. TN D-4384, NASA, Washington, DC, USA, 1968.

[3] C. Rogers, "Mainline Performance Prediction for Radial Inflow Turbine in Small High Pressure Ratio Turbine," VKI Lecture Series 1987-07, 1987.

[4] A. Whitfield and N. Baines, Design of Radial Turbomachines, John Wiley \& Sons, New York, NY, USA, 1990.

[5] H. Moustapha, M. F. Zeleski, N. C. Baines, and D. Japikse, Axial and Radial Turbines, Concepts NREC, White River Junction, Vt, USA, 2003.

[6] H. Aungier, Turbine Aerodynamics: Axial-Flow and RadialFlow Turbine Design and Analysis, ASME Press, New York, NY, USA, 2006.

[7] M. S. Y. Ebaid, F. S. Bhinder, and G. H. Khdairi, "A unified approach for designing a radial flow gas turbine," Journal of Turbomachinery, vol. 125, no. 3, pp. 598-606, 2003.
[8] C. A. Wasserbauer and A. J. Glassmann, "FORTRAN program for predicting off-design performance of radial-inflow turbines," Tech. Rep. TN D-8063, NASA, Washington, DC, USA, 1975.

[9] J. Harinck, Z. Alsalihi, J. P. Van Buijtenen, and R. A. Van den Braraembussche, "Optimization of a 3D radial turbine by means of an improved genetic algorithm," in Proceedings of the 6th European Conference on Turbomachinery, Fluid Dynamics and Thermodynamics, pp. 1033-1042, Lille, France, March 2005.

[10] A. Demeulenaere and Ch. Hirsh, "Application of multipoint optimization to the design of turbomachinery blades," in Proceedings of the 6th European Conference on Turbomachinery, Fluid Dynamics and Thermodynamics, pp. 1023-1032, Lille, France, March 2005.

[11] N. Binder, X. Carbonneau, and P. Chassaing, "Influence of a variable guide vane nozzle on the design parameters of a radial turbine stage," in Proceedings of the 6th European Conference on Turbomachinery, Fluid Dynamics and Thermodynamics, pp. 665-674, Lille France, March 2005.

[12] D. Japikse and N. C. Baines, Introduction to Turbomachinery, Concepts ETI, White River Junction, Vt, USA, 1994.

[13] B. Lakshminarayana, Fluid Dynamics and Heat Transfers of Turbomachinery, John Wiley \& Sons, New York, NY, USA, 1996.

[14] H. Chen and N. C. Baines, "Analytical optimization design of radial and mixed flow turbines," Proceedings of the Institution of Mechanical Engineers, Part A, vol. 206, no. 3, pp. 177-187, 1992.

[15] X. Carbonneau, M. Lemasson, and J. B. Cazalbou, "Contribution to the design of air-conditioning turbines," in Proceedings of the 13th International Symposium on Air Breathing Engines (ISABE '97), Chattanooga, Tenn, USA, September 1997.

[16] I. Watanabe, I. Ariga, and T. Mashimo, "Effect of dimensional parameters of impellers on performance characteristics of a radial inflow turbine," Journal of Engineering Power, pp. 81102, 1971. 

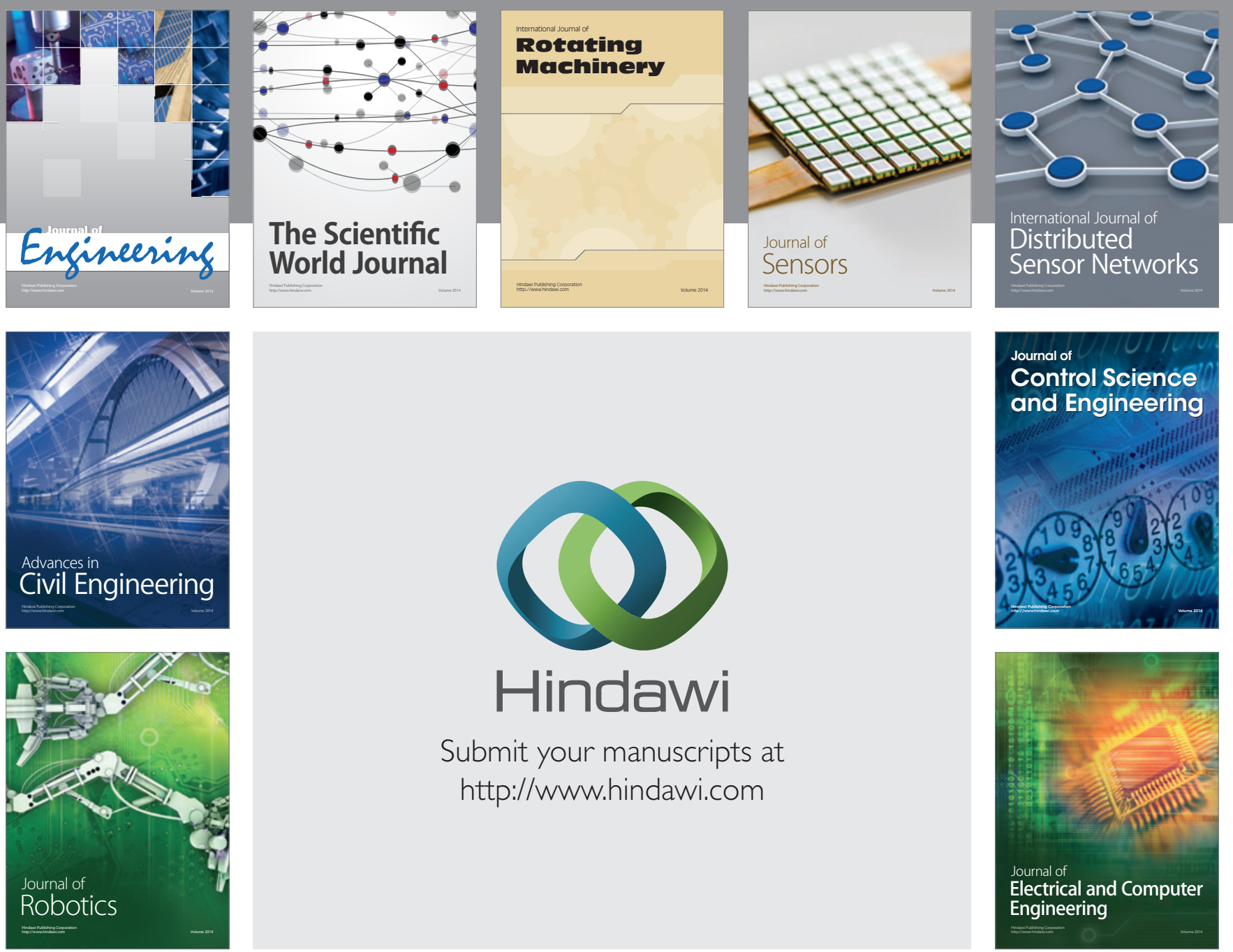

Submit your manuscripts at

http://www.hindawi.com
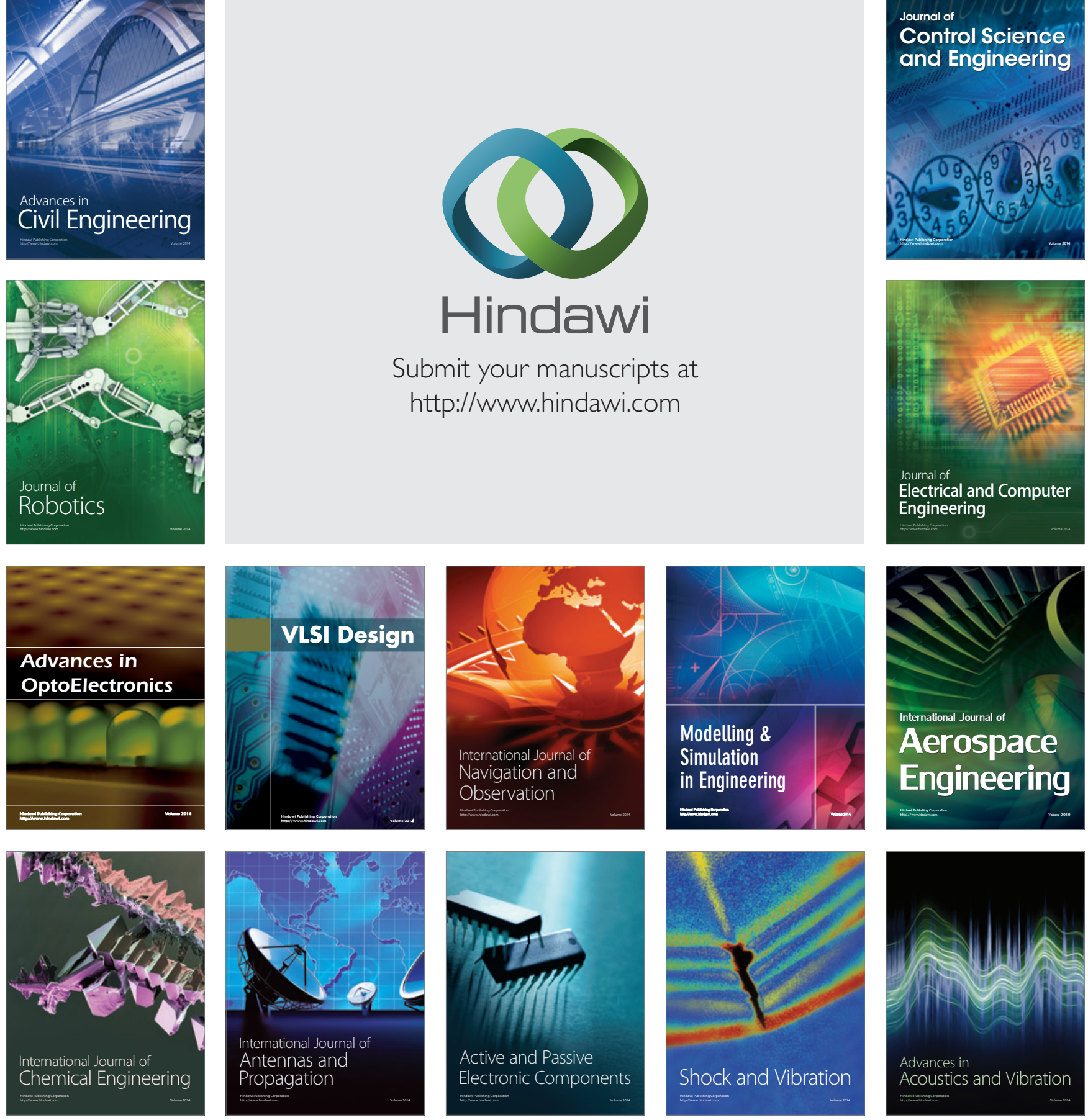